\title{
Assessment of Fatigue Resistance of Additivated Asphalt Concrete Incorporating Fibers and Polymers
}

\author{
Claudio Brovelli, Ph.D. ${ }^{1}$; Maurizio Crispino; Jorge C. Pais ${ }^{3}$; and Paulo A. A. Pereira ${ }^{4}$
}

\begin{abstract}
This paper reports the evaluation of fatigue response of asphalt mixtures produced with different additives, namely cellulose and synthetic fibers, amorphous polyolefin, and ethyl vinyl acetate (EVA), in comparison with conventional asphalt mixtures. The additive content was also analyzed by producing asphalt mixtures with 3,6, and 9\% of additive. Fatigue testing was performed with an indirect tensile test apparatus under controlled stress mode of loading. A comparative analysis of the fatigue resistance was carried out using the different methods. Fatigue life was defined using the classical approach in which the number of cycles reaches the double of initial deformation. It was also defined in terms of different methods based on dissipated energy: total dissipated energy, ratio of dissipated energy change, and plateau value. This paper offers an analysis of different methods of evaluating the indirect tensile fatigue test. The testing clearly shows that polymer modification may extend the fatigue life, and that energetic methods can be effectively applied to data from indirect tensile fatigue tests. DOI: 10.1061/(ASCE)MT.1943-5533.0000837. @ 2014 American Society of Civil Engineers.
\end{abstract}

Author keywords: Fatigue; Energy method; Additivated asphalt; Polymers; Fibers.

\section{Introduction}

Fatigue cracking is one of the main distress modes in asphalt pavements. It is essential to consider the loss of asphalt pavement serviceability in the design of asphalt mixtures and structural design of flexible pavements caused by the phenomenon of fatigue. Many laboratory test methods, such as the four-point bending test, two-point bending test, and indirect tensile tests, are available to characterize fatigue cracking of asphalt mixtures. In particular, the indirect tensile configuration is commonly adopted because of the possibility of using specimens compacted by both Marshall and shear gyratory compactor (Hartman et al. 2001) methods, as well as extracted from the road.

In most of cases, fatigue tests are carried out to evaluate the number of load cycles to reach the failure criterion, and the fatigue study of asphalt materials is conducted by assessing their fatigue curves (Wöhler curve). The choice of the failure criterion usually depends on the author or the paper, although most of the criteria used include the reaching of a certain percentage of initial stiffness (typically 50\%), the doubling of the initial deformation, or half of the initial stress (Lundstrom et al. 2004). This approach is generally considered as the traditional method to investigate fatigue behavior, and it is based on mathematical relationships between stress

${ }^{1}$ Politecnico di Milano, School of Civil and Environmental Engineering, 32 Piazza Leonardo Da Vinci, Milan 20133, Italy (corresponding author). E-mail: claudio.brovelli@mail.polimi.it

${ }^{2}$ Full Professor, Politecnico di Milano, School of Civil and Environmental Engineering, 32 Piazza Leonardo Da Vinci, Milan 20133, Italy. E-mail: maurizio.crispino@polimi.it

${ }^{3}$ Associate Professor, Univ. of Minho, Dept. of Civil Engineering, Guimarães 4800-058, Portugal. E-mail: jpais@ civil.uminho.pt

${ }^{4}$ Full Professor, Univ. of Minho, Dept. of Civil Engineering, Guimarães 4800-058, Portugal. E-mail: ppereira@ civil.uminho.pt

Note. This manuscript was submitted on September 26, 2012; approved on April 11, 2013; published online on April 13, 2013. Discussion period open until August 1, 2014; separate discussions must be submitted for individual papers. This technical note is part of the Journal of Materials in Civil Engineering, Vol. 26, No. 3, March 1, 2014. (C) ASCE, ISSN 08991561/2014/3-554-558/\$25.00. (or strain) and the number of loading cycles that the mixture needs to reach fatigue. Thus, the main disadvantage of this method is the lack of information in terms of fatigue behavior during the test; i.e., except for the stiffness reduction, no other information is obtained. In fact, the definitions of traditional fatigue resistance do not represent the internal state of the material, and they should be considered arbitrary.

One of the earliest attempts to characterize fatigue cracking in asphalt mixtures based on dissipated energy was proposed by Van Dijk and Visser (1977). This theory proposes the evaluation of the total cumulated energy spent by the material in controlled stress mode. The authors hypothesize that the total dissipated energy can be considered independent from the loading history. Van Dijk's theory was used by Ghuzlan and Carpenter (2000) to quantify fatigue based on the relative change in dissipated energy between consecutive cycles rather than the total energy from each cycle. In other words, the dissipated energy includes energy dissipated from viscoelasticity and energy dissipated from incremental damage during cyclic loading (Basin et al. 2009). For this reason, Carpenter and Shen (2006) proposed a method that considered the difference of the dissipated energy between two consecutive loading cycles, which indicates the amount of dissipated energy that produces the damage in the material (Ghuzlan and Carpenter 2000). These authors assumed that the material failure occurs in correspondence with a significant increase in the magnitude of the dissipated energy. The increment of dissipated energy is quantified through the ratio of dissipated energy change (RDEC).

Asphalt modification is widely used in road infrastructure to reduce fatigue cracking and rutting phenomena. The best-known procedure of binder improvement is by means of polymer modification. Therefore, some types of fiber are often added to asphalt mixtures. The effects on binder are dependent on the modifier, even if the general improvements include changes in the viscoelasticity of binder, increases in dynamic modulus, reduction in moisture susceptibility, rutting resistance, freeze-thaw, and fatigue resistance. Thus, this paper assesses the indirect tensile fatigue resistance of different asphalt mixtures with cellulose and synthetic fibers, amorphous polyolefin, and a particular polymer obtained as compound of low-density polyethylene (LDPE) and ethyl vinyl acetate 
(EVA). The fatigue resistance was evaluated according to the plateau value (PV) of the ratio of dissipated energy change (RDEC).

\section{Materials and Testing Procedures}

\section{Selection of Materials}

In this study, four different asphalt mixtures containing (1) no additives (BO mixture), (2) cellulose and synthetic fibers (BCS mixtures), (3) amorphous polyolefin (named P1) (BPI mixtures), and (4) a particular polymer obtained as a compound of LDPE and EVA (named P2) (BPS mixtures) were evaluated in terms of fatigue resistance using the plateau value (PV) approach.

Mixture design was performed according to the national design procedure proposed by the Italian specifications for binder course (25-mm maximum aggregate size) and included ordinary bitumen ( $85 \mathrm{~mm} / 10$ penetration, $44^{\circ} \mathrm{C}$ softening point) and virgin aggregates. The common composition of all asphalt mixtures consisted of $4.1 \%$ of optimum binder content by mass of total asphalt mixture and $3.9 \%$ of optimum (target) void content.

The addition of polymers into the asphalt mixture is not so common in Italy, unlike modified bitumen with polymers. Therefore, for this research, both P1 and P2 were provided as small pellets, workable at room temperature, so they could be added directly into the mixture just before hot bitumen. The cellulose and synthetic fibers were obtained in the form of a bundle of textile threads, insoluble in water, bulk density between 0.45 and $0.55 \mathrm{~kg} / \mathrm{cm}^{3}$, and with a diameter between 4 and $6 \mu \mathrm{m}$ (black colored). The thread lengths varied between 5 and $8 \mathrm{~mm}$. Three additive contents were considered: 3,6 , and $9 \%$ by weight of bitumen. Thus, a total of 10 mixtures were studied. For each additive, a minimum of six specimens was compacted, so a total of 60 samples were tested.

Air voids were not consistent because mixtures were all compacted by means of the same compaction protocol (Superpave mix design) and they were modified using very different additives. In particular, the fluidifying effect of polymers (even at 3\%) caused an overcompaction of the material in the mold, resulting in lower voids (air). Specimens from mixtures with P1 and P2 were affected by different volumetric characteristics. Nevertheless, based on presented results, it is difficult to establish that the differences within mixtures are the result of primarily volumetric changes. In this sense, the gap between modified and unmodified mixtures was only increased. Fatigue resistance is known to depend on volumetric characteristics. Nevertheless, the present research aimed at a different comparison. All mixtures were carried out at the same compaction energy instead of at constant volumetric parameters; thus, the differences in void content were not taken into consideration in the fatigue analysis.

\section{Specimen Preparation}

All mixtures containing bitumen, fibers, and P1 were mixed and compacted at $140^{\circ} \mathrm{C}$ independently from the additive content. In contrast, mixtures with $\mathrm{P} 2$ were mixed at $175^{\circ} \mathrm{C}$ and compacted at $140^{\circ} \mathrm{C}$ because of the highest melting point of the added polymer. Each cylindrical specimen had a dimension of $150 \mathrm{~mm} \times 50 \pm 5 \mathrm{~mm}$, obtained from a gyratory shear compactor in agreement with the Superpave mixture design procedure.

\section{Fatigue Test Procedure}

An indirect tensile fatigue test (ITFT) was used. This test follows the European Standard EN 12697-24. Testing was controlled by load so that the maximum specimen stress could remain constant while the displacement induced by the load increased at every cycle during the test at $20^{\circ} \mathrm{C}$. The shape of the loading wave was haversine, without rest periods, and its frequency was $2 \mathrm{~Hz}$. Fatigue was assumed to have been reached and the test completed when the resulting strain $\left(\varepsilon_{\text {fatigue }}\right)$ was two times the initial strain $\left(\varepsilon_{0}\right)$ (computed considering the initial value of the one obtained at cycle 100).

\section{Evaluation of Fatigue Resistance}

\section{Fatigue Curves}

A traditional fatigue analysis was performed using the data collected according to EN 12697-24 procedures for fatigue tests. The number of cycles to failure $\left(N_{f}\right)$ was defined as the number of load repetitions when the deformation was twice the initial value. The fatigue results for the studied asphalt mixtures are presented in Table 1.

As indicated in Table 1, the results show how the addition of both P1 and P2 induced a significant increase in fatigue resistance, quantified by the increment of $N_{f}$. The results also indicate that the increase in additive content increases the fatigue life that is expressed in terms of the increase in the $k_{1}$ coefficient. The increase of the fatigue life after increasing the additive content is extremely high, which clearly reveals the benefit of using additives in asphalt mixtures. In contrast, the variation of coefficient $k_{2}$ is limited because it is related primarily with the type of asphalt mixture and not with the performance of the binder.

With the decrease in the stress level, the role of additives in fatigue resistance remains. Except for fiber-modified mixtures, the ranking between mixtures was insensitive to the stress level (Fig. 1). However, decreasing from 500 to $300 \mathrm{kPa}$ made the number of cycles increase until two orders of magnitude. In particular, the load cycles to failure reach the maximum for mixtures PI6 and PI9 at $300 \mathrm{kPa}$ and also a minimum for those containing fibers and no additives (unmodified). As shown in Fig. 1, even for lower dosages of polymers (both P1 or P2), the number of cycles to failure increased significantly. Nevertheless, mixtures containing cellulose and synthetic fibers showed different behaviors by changing the stress level even with a slight increase in cycles to failure. At $300 \mathrm{kPa}$, the number of cycles did not change significantly with the dosage of fibers, whereas at higher stress levels the influence of additives was more evident.

The fatigue properties of the materials may vary significantly at a given time and temperature. In particular, for modified asphalt binders, the rheological behavior of the mixture is strongly influenced by the nature of the modifier. Thus, fatigue tests carried out on asphalt mixtures with different stiffness show different deterioration paths and different critical levels of stiffness at failure. For mixtures

Table 1. Results from Fatigue Tests: Traditional Analysis

\begin{tabular}{|c|c|c|c|c|c|c|c|}
\hline \multirow[b]{2}{*}{ ID } & \multicolumn{2}{|c|}{$N_{f}$} & \multirow{2}{*}{$\begin{array}{c}\text { Intercept } \\
\left(k_{1}\right)\end{array}$} & \multirow{2}{*}{$\begin{array}{c}\text { Slope } \\
\left(k_{2}\right)\end{array}$} & \multirow[b]{2}{*}{$R^{2}$} & \multicolumn{2}{|c|}{ Standard deviation } \\
\hline & $300 \mathrm{kPa}$ & $500 \mathrm{kPa}$ & & & & $300 \mathrm{kPa}$ & $500 \mathrm{kPa}$ \\
\hline $\mathrm{BO}$ & $5.8 \times 10^{2}$ & $2.2 \times 10^{2}$ & 60.419 & 1.880 & 0.99 & 42.4 & 2.8 \\
\hline $\mathrm{BCS} 3$ & $1.8 \times 10^{3}$ & $3.4 \times 10^{2}$ & 1.759 & 5.945 & 0.99 & 28.2 & 24.7 \\
\hline BCS6 & $1.8 \times 10^{3}$ & $2.0 \times 10^{2}$ & 9.105 & 4.409 & 0.97 & 43.8 & 55.9 \\
\hline BCS9 & $2.3 \times 10^{3}$ & $1.1 \times 10^{2}$ & 34.548 & 3.286 & 0.99 & 67.2 & 57.2 \\
\hline BPI3 & $2.1 \times 10^{4}$ & $8.6 \times 10^{2}$ & 4.070 & 7.102 & 0.86 & 170.9 & 63.2 \\
\hline BPI6 & $1.7 \times 10^{5}$ & $1.9 \times 10^{4}$ & 923.218 & 4.355 & 0.98 & 600.3 & 455.9 \\
\hline BPI9 & $2.5 \times 10^{5}$ & $2.8 \times 10^{4}$ & 566.609 & 5.184 & 0.88 & 690.1 & 211.4 \\
\hline BPS3 & $2.3 \times 10^{3}$ & $5.4 \times 10^{2}$ & 57.396 & 3.088 & 0.88 & 131.3 & 17.7 \\
\hline BPS6 & $2.5 \times 10^{4}$ & $4.7 \times 10^{3}$ & 231.479 & 3.953 & 0.79 & 161.5 & 190.1 \\
\hline BPS9 & $1.2 \times 10^{5}$ & $2.2 \times 10^{4}$ & 685.181 & 4.476 & 0.83 & 170.4 & 96.8 \\
\hline
\end{tabular}



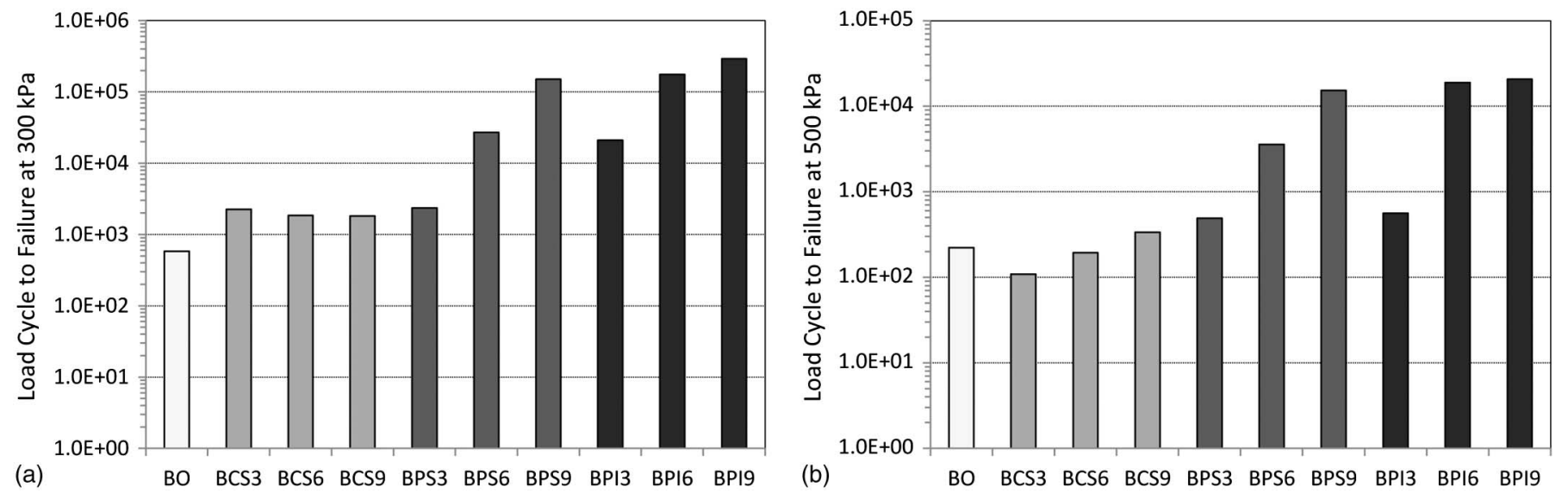

Fig. 1. Mixtures ranking on load cycle to failure at (a) $300 \mathrm{kPa}$; (b) $500 \mathrm{kPa}$

containing unmodified binder and fibers, the decrease in stiffness was not noticeable, as shown by mixtures containing polymers [Figs. 2(a and b)]. For the stiffest modified mixtures containing $\mathrm{P} 1$ and $\mathrm{P} 2$, the decrease in stiffness was smaller compared with the unmodified ones, and it did not decrease more than $10 \%$ before failure except for mixtures BPS6 and BPI3 [Figs. 2(c and d)].

An important observation from the tests carried out in this study is that the stiffness decrease rate is always diminishing in all mixtures. Di Benedetto et al. (2004) demonstrated the existence of four main typical paths for stiffness during a fatigue test. Each of them related to the type of loading and uniformity of strain inside the specimens. They classified these paths using the slope and the shape of the stiffness curves at different loading cycles. According to their theory, in stress-controlled tests, the stiffness decreases in cycles until complete failure. For this reason, the failure is easily identified and a percentage of the stiffness decrease may be used as a failure criterion [Figs. 2(a and b)]. This is the case of unmodified mixtures and mixtures with fibers, in which the stiffness modulus keeps decreasing until failure. In contrast, mixtures with polymers showed a smaller stiffness decrease because of the highest stiffness and the absence of decay in the performances during loading cycles.

\section{Total Dissipated Energy $\left(W_{\text {tot }}\right)$}

Fig. 3 compares the performance of the four mixtures tested for all additive contents. A material with a greater magnitude of dissipated
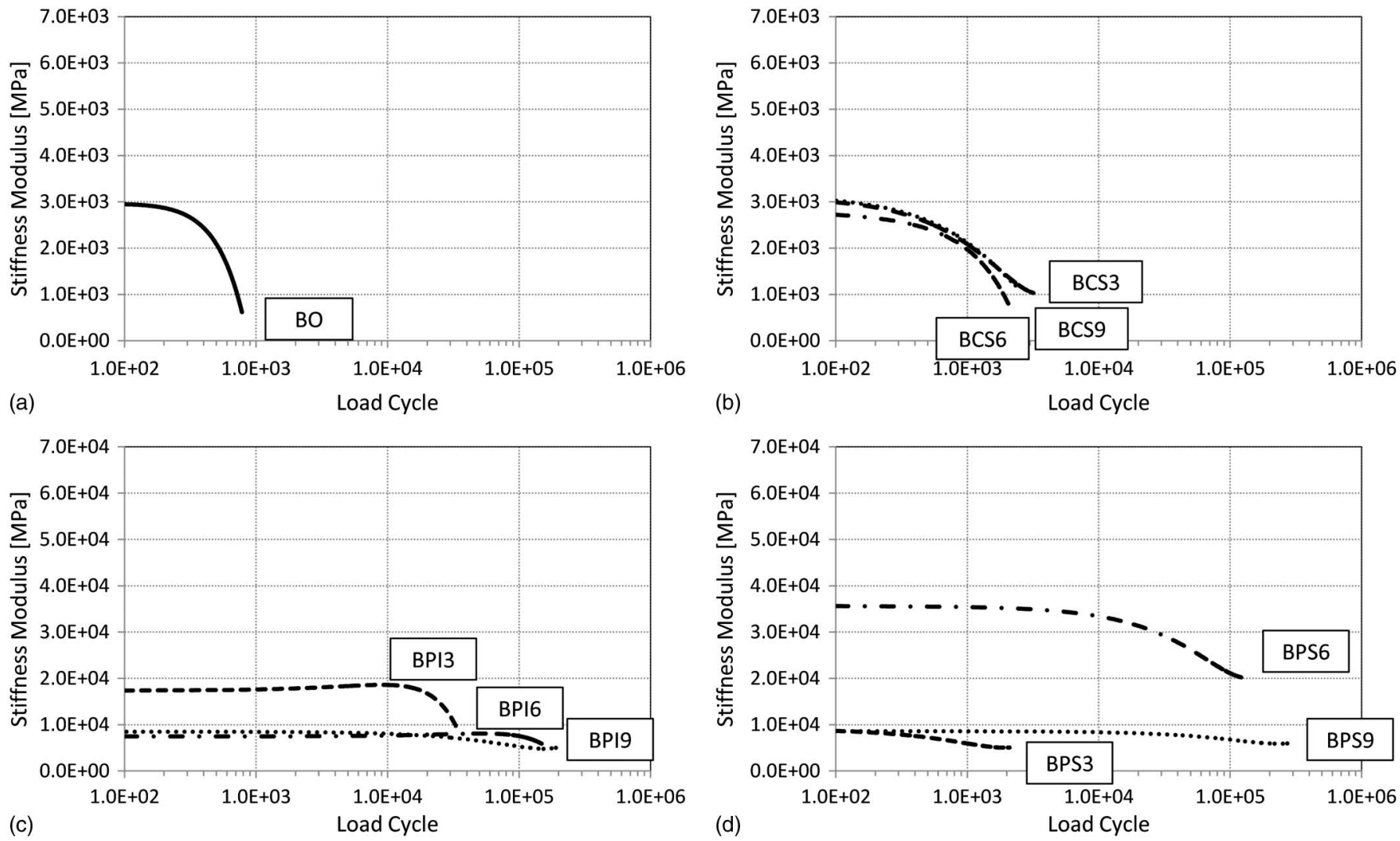

Fig. 2. Stiffness evolution of studied mixtures: (a) BO mixture; (b) BCS mixtures; (c) BPI mixtures; (d) BPS mixtures 


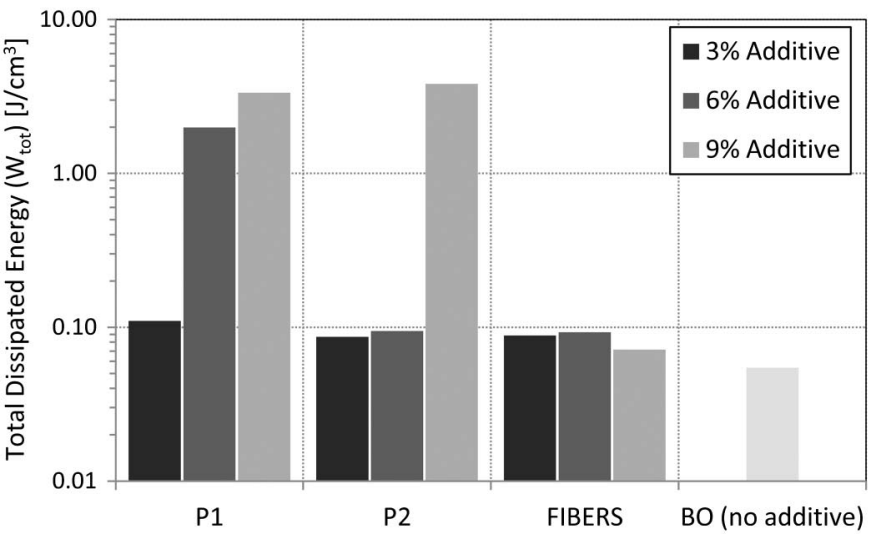

Fig. 3. Total dissipated energy ( $\left.W_{\text {tot }}\right)$ of all mixtures: ITFT at $20^{\circ} \mathrm{C}$, controlled-stress at $300 \mathrm{kPa}$

energy until it reaches failure is more resistant to fatigue cracking. Basin et al. (2009) suggested that the total dissipated energy is sensitive to the mode of loading, to the amplitude of strain or stress for any given mode of loading. The inconsistency in performance ranking of different mixtures and lack of constancy in results from different modes of loading can be partially attributed to the fact that the total dissipated energy includes viscoelastic energy that does not come from incremental fatigue damage.

Fig. 4 compares the values of the phase angle at cycle 100 . Mixtures with high $W_{\text {tot }}$ also clearly showed great values of phase angle. Sensitive variations on the phase angle indicate mixtures with different viscoelastic behavior. The apparent phase angle determined experimentally from the response of a cyclic fatigue test is the sum of the true viscoelastic phase angle (resulting from the viscoelastic deformation) and the apparent increase in phase angle resulting from damage accumulated during the load cycle. In fatigue tests, this indicates that the computation of $W_{\text {tot }}$ is affected by the viscoelastic component.

Mixtures containing P1 and P2 showed the best results in terms of $W_{\text {tot }}$. The presence of polymers led to an increment on $W_{\text {tot }}$. In particular, the amount of total energy at failure was similar for low and high additive contents, although it showed a sensitive variability for intermediate values. In contrast, fibers showed no significant variation in the behavior of the mixture.

\section{Dissipated Energy (DE)}

The evaluation of the dissipated energy (DE) through a regression function can give the rate of approximation of the energetic

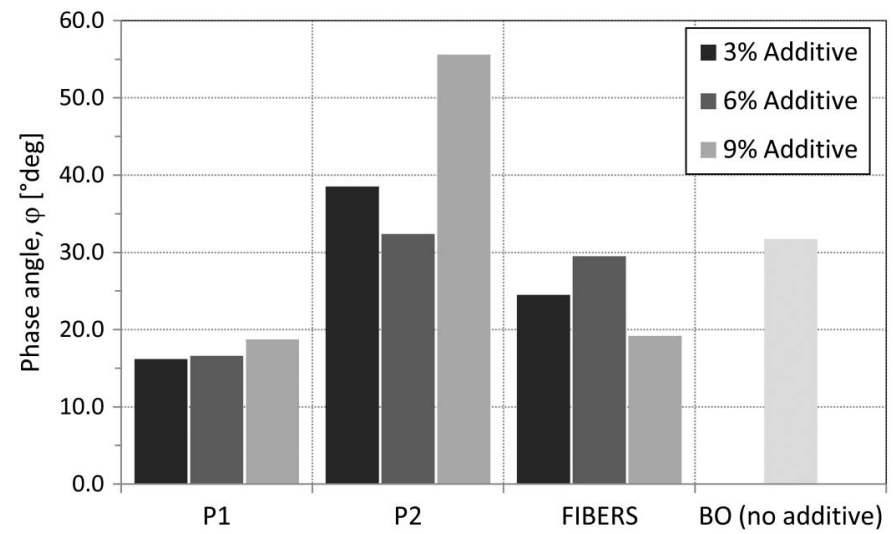

Fig. 4. Phase angle of all mixtures tested at $300 \mathrm{kPa}$ behavior of each mixture. For tested mixtures, the DE versus cycles was plotted through a regression curve using the power law form.

As recommended by Shen and Carpenter (2007), the initial segment (initial load repetitions) of the DE-LC curve was not taken into account. The same authors suggested extending the bending beam fatigue tests up to 1 to 8 million loading cycles when investigating the fatigue endurance limit (FEL).

In the present work, all mixtures failed before reaching $10^{6}$ cycles, particularly for tests at $500 \mathrm{kPa}$, in which tested specimens failed after a few thousand cycles. Thus, the corresponding DE-LC curves were discharged because of the lack of points. In contrast, the test duration could be increased by reducing the stress to $300 \mathrm{kPa}$, obtaining more data to fit the DE-LC curve.

In the present research, all fatigue tests were conducted in controlled stress in indirect tensile configuration. For controlled stress, DE tends to increase with the loading cycle because of the increase in deformation during the test, as demonstrated by the definition of DE. The opposite trend is registered for controlled strain (stress decreases). For this reason, the power law relationship did not match the expected requirement of accuracy and $R^{2}$. To overcome this problem, the exponential form of the DE-LC curve was chosen. The exponential model was observed to always produce the best $R^{2}$ when compared with the power law.

\section{Plateau Value (PV)}

For a controlled stress (or strain) fatigue test, lower stress (or strain) levels correspond to longer fatigue life of a mixture. Regardless, many studies have demonstrated that there is not a unique relationship between stress or strain levels and fatigue life for different mixture types, which becomes a disadvantage of the traditional fatigue analysis approach (Shen and Carpenter 2005). The correlation between PV and $N_{f}$ removes this ambiguity, as a lower PV always corresponds to a longer $N_{f}$.

A change in damage accumulation results in a consistent corresponding change in load repetitions to failure. This relationship indicates that the energy dissipation mechanism of the mixture was consistent over all levels of energy input, resulting in a unique relationship with loads to failure $\left(\mathrm{PV}-N_{f}\right)$. As demonstrated by Carpenter et al. (2003), the relation between load cycle and PV in bending beam fatigue tests is well known. Fig. 5 suggests that the same dependence may exist in indirect tension fatigue tests. The data points were well distributed along the regression line, indicating a reasonable prediction. Thus, mixtures with P1 showed the lowest PV, whereas unmodified mixtures (BO) and mixtures containing fibers showed higher values. This confirms that independently of the mixture, the fatigue behavior can be efficiently described through the energetic theory of dissipated energy and

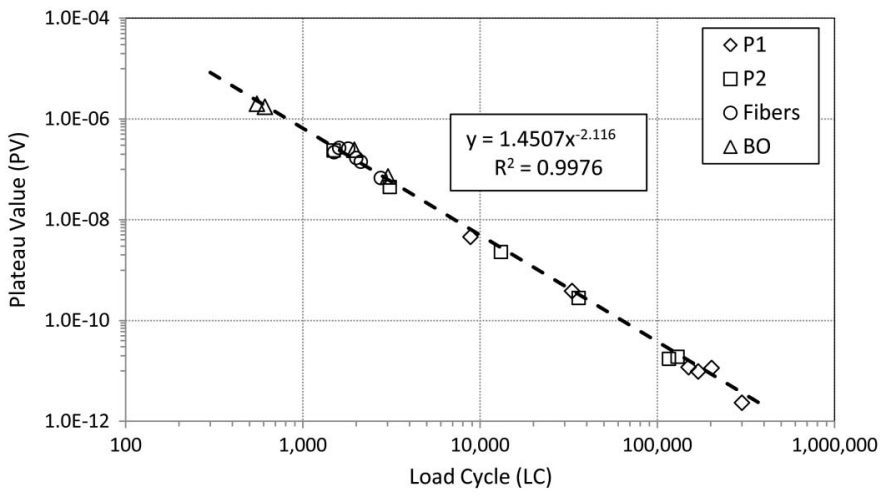

Fig. 5. Comparison of plateau value (PV) for all mixtures 
PV. In addition, the consistency of results in Fig. 5 confirms the importance of $\mathrm{PV}$ as an indicator of fatigue resistance.

Considering the behavior of the mixtures, the mixtures containing fibers were grouped in a restricted area, whereas mixtures with polymers showed a sensitive variation on PV that depended on the additive content. In particular, mixtures containing P1 covered the widest range.

\section{Conclusions}

The indirect tensile fatigue tests conducted in controlled stress at $20^{\circ} \mathrm{C}$ to investigate the behavior of four mixtures including fibers and polymers modified asphalt revealed the following:

1. The classical fatigue approach used in this study uniquely concerns the number of cycles to failure. Consequently, single mixtures may exhibit different fatigue behavior, viscoelastic response to failure, but still no other information apart from load cycle can be obtained;

2. The analysis about total energy $\left(W_{\text {tot }}\right)$ and phase angle was useful as an initial estimation of the dissipated energy in the fatigue damage process. Therefore, this approach hid the effective contribution to failure from fraction resulting from viscoelastic energy dissipation;

3. The results presented in this paper show that energetic theories can be effectively applied to indirect tensile fatigue tests (ITFT). When the stress applied is constant (controlled stress), it is recommended to compute DE-LC using an exponential law relationship. This criterion was empirically established and, consequently, requires many tests to be performed;

4. The PV represents a good indicator for fatigue resistance. High values of $R^{2}$ demonstrate the existence of a unique curve of $\mathrm{PV}-N_{f}$, also in ITFT configuration for the tested condition of stress and temperature; and

5. To deepen the healing phenomenon, an in-depth fatiguehealing study is required, e.g., the self-restoration of asphalt during resting periods or the ability of bitumen to recover from deformation when unloaded.

In contrast, the following conclusions on mixtures can be drawn:

1. The addition of polymers results in a significant improvement on fatigue life. From reported results, these benefits can be quantified using both classic approaches $\left(N_{f}\right)$ and energetic methods (DE, PV);

2. An increment in additive contents always corresponds to a better fatigue resistance. In particular, mixtures containing P1 showed the best results in terms of fatigue life;

3. The results obtained in this work revealed that the use of $3 \%$ of polymer P1 and P2 as well as fibers do not improve the fatigue life of asphalt mixtures; and

4. The use of 6 and $9 \%$ of polymer P1 and P2 as well as fibers improved the fatigue resistance of asphalt mixtures.

\section{References}

Basin, A., Castelo Branco, V. T. F., Masad, E., Little, D. N. (2009). "Quantitative comparison of energy methods to characterize fatigue in asphalt materials." J. Mater. Civ. Eng., 10.1061/(ASCE)08991561(2009)21:2(83), 83-92.

Carpenter, S. H., Ghuzlan, K., and Shen, S. (2003). "A fatigue endurance limit for highway and airport pavements." J. Transport. Res. Board, 1832(1), 131-138.

Carpenter, S. H., and Shen, S. (2006). "Dissipated energy approach to study hot-mixture asphalt healing in fatigue." J. Transport. Res. Board, 1970, $178-185$.

Di Benedetto, H., De La Roche, C., Baaj, H., Pronk, A., and Lundstrom, R. (2004). "Fatigue of bituminous mixtures." Mater. Struct., 37(3), 202-216.

Ghuzlan, K. A., and Carpenter, S. H. (2000). "Energy-derived, damage based failure criterion for fatigue testing." J. Transport. Res. Board, 1723, 141-149.

Hartman, A. M., Gilchrist, M. D., and Walsh, G. (2001). "Effect of mixture compaction on indirect tensile stiffness and fatigue." J. Transp. Eng., 10.1061/(ASCE)0733-947X(2001)127:5(370), 370-378.

Lundstrom, R., Di Benedetto, H., and Isacsson, U. (2004). "Influence of asphalt mixture stiffness on fatigue failure." J. Mater. Civ. Eng., 10.1061/(ASCE)0899-1561(2004)16:6(516), 516-525.

Shen, S., and Carpenter, S. H. (2005). "Application of the dissipated energy concept in fatigue endurance limit testing." J. Transport. Res. Board, 1929, 165-173.

Shen, S., and Carpenter, S. H. (2007). "An energy approach for airport pavement low damage fatigue behavior." Proc., FAA Worldwide Airport Technology Transfer Conf., Atlantic City, NJ.

Van Dijk, W., and Visser, W. (1977). "The energy approach to fatigue for pavement design.” J. Association of Asphalt Paving Technologists, 38, $1-40$. 